\title{
Study on the optimal scheduling of emergency resources for hazardous material incidents caused by human factors
}

\author{
Wang Sheng \\ Shanghai maritime university Transportation College, Shanghai Pudong new area, 1550 Harbour Avenue, \\ 201306 \\ E-mail:18217315929@163.com
}

Keywords: Hazardous material incidents, Emergency resource scheduling, Human factors

\begin{abstract}
The emergency resource scheduling of hazardous material incidents involves many problems, which affect the people's life and property safety. On the one hand, the paper analyzed the causes of hazardous material incidents caused by human factors; on the other hand, it put forward corresponding emergency resource scheduling schemes based on ideal single accident point models and multi-accident points and multi-resource models, which prevent hazardous material incidents from aggravating and spreading during the rescue. The paper provides a reference for the related research on emergency resource scheduling of hazardous material incidents caused by human factors.
\end{abstract}

\section{Introduction}

With the development of industrialization in China, the production and transportation of hazardous materials are increasing rapidly each year, which raised the number of accidents at the same time. Then, the author advised to make a brief account of the emergency resource scheduling research on hazardous materials at home and abroad.

Zhai Huaihai et al. have studied the emergency evacuation transportation problem of hazardous materials in different situations ${ }^{[1]}$; The relevant departments of the state have done a lot of research on public events, natural disasters, chemical spills and other emergencies, and provided more than 200 contingency plans ${ }^{[2]}$. Ye Long has studied the emergency dispatch of offshore oil spill, and constructed the optimal scheduling model with the aim of the shortest emergency time and the least emergency resource point with combining with the characteristics of emergency resources. ${ }^{[3]}$

Besides, Haghan et al. regarded the emergency logistics as a multi-variety and multi-mode network flow problem with time window limit ${ }^{[4]}$; Fiedrich et al. have improved the quality of rescue and minimized the number of deaths by using resources efficiently under time, resource quantity and quality of resources are limited, which gave multiple accident point distribution and transport of goods of optimal model after earthquake ${ }^{[5]}$.

\section{Hazardous material incidents caused by human factors}

There are many reasons for the occurrence of hazardous material incidents, which can be divided into human factors and non-human factors. The non-human factors is determined by the nature of hazardous material, roads, the weather and other reasons. To some extent, the accident is inevitable. However, hazardous material incidents caused by human factors can be avoided by means of certain preventive measures. The causes of hazardous material incidents caused by human factors are as follows:

(1) Driver fatigue driving, or random traffic violation.

(2) Insufficient understanding of the physical and chemical properties of transported hazardous materials.

(3) The safety awareness of the hazardous materials driver is not enough, and the importance of the transport of hazardous materials is not fully understood, and the driver's sense of responsibility is too poor. 
(4) The relevant laws and regulations on hazardous materials are not perfect enough so that there are some omissions and loopholes in the management of hazardous materials.

(5) The decision makers make the wrong judgment or issue the wrong instruction, which make the accident worse.

The reason of hazardous material incidents is largely determined by the low quality of drivers, so it is very important to strengthen the training of drivers' personal qualities. In addition, the relevant laws and regulations are suggested to improve to investigate the legal responsibility of the relevant personnel. Finally, it's necessary to strengthen managers' ability to make decisions and find the best solution to make emergency dispatch time and rescue point at least.

\section{Study on optimization of emergency resource scheduling for the ideal single accident point and multi-accident point and multi-resource hazardous materials}

\subsection{Ideal single accident point emergency resource scheduling}

First of all, we are advised to discuss the single accident point problem, namely, there is only one accident spot in the region. Assuming the road was smooth and would not affect the emergency resource scheduling time due to traffic jams and other road conditions; It is also assumed that the emergency resources required for the accident were limited, that is, the total amount of emergency resources required was determined. The primary purpose of the rescue of dangerous goods accident is to rescue as soon as possible, in order to minimize the casualties and property losses caused by the accident. Since there may be regenerative accidents in the rescue process of hazardous material incidents, it is also necessary to consider the stability of hazardous material resource scheduling system. To sum up, the emergency resource scheduling of hazardous material should meet two goals; 1 . timeliness of emergency resource scheduling: namely, the time of emergency resource scheduling $(\mathrm{T})$ is the shortest; 2. stability of emergency resource scheduling system: that is, the number of rescue points is the least.

\subsection{Multi-Accidents and Multi-resource emergency resource scheduling}

The emergency scheduling scheme should make the scheduling of emergency resources as short as possible. However, there is a tolerance for the scheduling time of emergency resources. In the range of time tolerance of emergency resource scheduling, the degree of satisfaction can be considered to be 1 . If it exceeds a certain range, with the extension of time, the satisfaction degree of emergency resource scheduling decreases gradually until 0 . The relationship between scheduling time and satisfaction degree of emergency resources can be expressed by coordinate axis graph. The horizontal axis represents the emergency scheduling time, and the vertical axis represents the satisfaction, as shown in Figure 1: The constraint satisfaction function of the emergency scheduling time is shown in Figure 2:

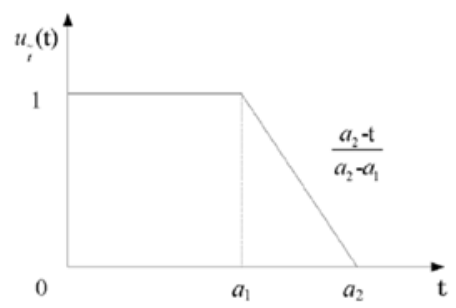

Figure 1 Relationship between emergency scheduling time and satisfaction

$$
u_{t}(\mathrm{t})= \begin{cases}1, & 0 \leq \mathrm{t} \leq a_{1} \\ \frac{a_{2}-\mathrm{t}}{a_{2}-a_{1}}, & a_{1} \leq \mathrm{t} \leq a_{2} \\ 0, & \mathrm{t} \geq a_{2}\end{cases}
$$

Figure 2 emergency scheduling time and constraint satisfaction function 
The more the number of rescue points, the lower the satisfaction of emergency scheduling is. Assuming an extreme situation, where there was no accident, the number of rescue points was zero, and the emergency scheduling satisfaction was 1 . However, when all the rescue sites are involved in the rescue work, the satisfaction of emergency scheduling is $0^{[6]}$.

The method of establishing the membership function of two fuzzy sets is given by Yang lunbiao ${ }^{[7]}$. According to the above two constraint satisfaction functions mentioned above, the membership function of any rescue scheme $\varphi$ is defined as $u(\varphi)$, and its expression is shown in Figure 3. The $\lambda$ is the decision preference of the decision makers, here we need to make a special description of $\lambda$ : Taking casualties at least as the goal, to take into account the least number of casualties by the means of analyzing and judging multiple accidents. This chapter focuses on the decision makers to make corresponding decisions in terms of casualties, with the minimal casualties as the main relief purpose.

$$
\operatorname{Max} \quad u(\Phi)=\lambda u_{\sim}(\mathrm{T}(\Phi))+(1-\lambda) f_{\sim}(\Phi) \quad 0<\lambda<1
$$

Figure 3: membership function $\mathrm{u}(\Phi)$

However, in consideration of many accidents being located in places with different crowd density, the casualties and losses caused by the accidents being very different, a scheduling rule based on casualties is adopted. The specific ideas are as follows: first of all, to classify emergency resources and scheduling resources preferentially when resources required for only one of the incident sites. The common resources are scheduled according to the size of the accident and the casualties caused by the accident. The degree of each accident casualties is carried outed from small to large, then neighborhood search algorithm is used to obtain emergency rescue scheme. Finally, the emergency resource scheduling scheme of multiple accident points can be obtained.

\section{Conclusions}

The paper has analyzed the causes of hazardous material incidents caused by human factors; it has put forward corresponding emergency resource scheduling schemes based on ideal single accident point models and multi-accident points and multi-resource models, which prevent hazardous material incidents from aggravating and spreading during the rescue. The paper provides a reference for the related research on emergency resource scheduling of hazardous material incidents caused by human factors.

\section{Reference}

[1] Zhai Huaihai. A shallow exploration of urban population emergency evacuation transportation [J]. China emergency rescue, 2007, (3): 14-15.

[2] Jiang Yunzhao, Zhu Wanghong, Qiu Guoqing, et al. Study on evacuation of personnel under the influence of earthquake secondary disasters [J]. China safety production science and technology, 2008, 4(1): 38-41.

[3] Ye Long. Research on the development and development of emergency resource transfer system for offshore ships based on GIS [D]. Shanghai maritime university, 2006.

[4] SHENG-XUE HE, FAN B Q. Searching the effective paths in dynamic transportation network [J]. Journal of University of Shang reference.

Hai for Science \& Technology, 2006.

[5] FIEDRICH F, GEHBAUER F, RICKERS U. Optimized resource allocation for emergency response after earthquake [J]. Safety Science, 2000, 35(1): 41-57.

[6] Yin Hui. Study on optimal scheduling of emergency resources for hazardous materials in uncertain situations [D]. South China university of technology, 2013.

[7] Yang Yanyu, Gao Yingyi. Fuzzy mathematics: principles and applications [J], 2003. 